Jaime Borrego-Jiménez ${ }^{1}$ Claudia Soria-Segarra ${ }^{2}$ Teodora Diana Moldovan ${ }^{3}$ José María Navarro-Mari' José Gutiérrez-Fernández ${ }^{1,3}$

\title{
Use of WalkAway MicroScan system colistin well when determining the susceptibility of Pseudomonas aeruginosa and Acinetobacter baumannii recent clinical isolates
}

\author{
${ }^{1}$ Departamento de Microbiología, Hospital Universitario Virgen de las Nieves-Instituto de Investigación Biosanitaria de \\ Granada. Granada. \\ ${ }^{2}$ Departamento de Microbiología. Facultad de Ciencias Médicas, Universidad de Guayaquil, PC, EC090112, Ecuador. \\ ${ }^{3}$ Departamento de Microbiología, Facultad de Medicina, Universidad de Granada- Instituto de Investigación Biosanitaria \\ de Granada. Granada.
}

\section{Article history}

Received: 7 October 2019; Revision Requested: 29 October 2019; Revision Received: 3 November 2019; Accepted: 5 November 2019; Published: 2 January 2020

\section{Sir,}

The increasing number of multiresistant non-fermenter Gram-negative bacilli isolates has boosted the use of antibiotics, like colistin, for the treatment of the infections caused by these microorganisms [1]. Unfortunately, the assessment of the colistin ssusceptibility is not an easy task in a microbiology laboratory, thus finding a solution for this situation has become essential. It has been described that disc-diffusion and E-test methods are not reliable tools, since a high percentage of false resistance has been detected as their result [2]. Based on the EUCAST guidelines [3], the gold standard for the colistin sensitivity determination would be the broth microdilution method, even though this implies the added difficulty of the manual processing. A few alternatives can be considered, like the Sensititre (ThermoFisher Diagnostics, U.S.) and the MicroScan (Beckman Coulter, U.S.) panels but, even in these, the several performed studies have found that false resistances can appear in up to $65 \%$ of the cases [4]. Another useful method, considering the EUCAST's proposal, is the manual microdilution used in UMic test (BioCentric, France), which we are currently using in our laboratory in order to confirm the resistances that the MicroScan system has previously found. The aim of our study was to compare the automatized MicroScan and E-test (gradient diffusion) methods to the UMic manual microdilution.

A total of 23 isolates (17 Pseudomonas aeruginosa and 6 Acinetobacter baumannii), all proceeding from urine cultures of different clinical episodes, have been studied between April 2018 and April 2019, being all interpreted as colistin-resistant by the MicroScan system (table 1). Focusing our attention on the study of false positives (major errors), colistin suscep-

Correspondence:

José Gutiérrez-Fernández.

Laboratorio de Microbiología. Hospital Universitario Virgen de las Nieves.

Avenida de las Fuerzas Armadas, 2. E-18012 Granada, España.

E-mail: josegf@go.ugr.es tibility has been evaluated using the gradient-diffusion E-test (MIC Strip, Liofilchem, Italy) method in Müller-Hinton agar (BD, Spain) and through microdilution (UMic), which was used as the reference method. The colistin susceptibility study was carried out accordig to the manufacturers' instructions. The ATCC 27853 (American Type Culture Collection) strain of Pseudomonas aeruginosa was used as control.

The possible MIC ranges were $\leq 0.06$ to $>64 \mathrm{mg} / \mathrm{L}$ for the manual microdilution, $\leq 2$ to $>4 \mathrm{mg} / \mathrm{L}$ for the MicroScan system and $\leq 0.016$ to $>256 \mathrm{mg} / \mathrm{L}$ corresponding to the E-test method. The obtained results are presented in the table 1. A MIC $>2 \mathrm{mg} / \mathrm{L}$ was obtained in 7 isolates (30.4\% of the samples) through E-test method, in 4 isolates (17.4\%) through manual microdilution and in 2 isolates (8.7\%) using both techniques. A total of $83 \%$ of the isolates that MicroScan system identified as having a MIC $\geq 4 \mathrm{mg} / \mathrm{L}$ resulted to be susceptible (MIC $\leq$ $2 \mathrm{mg} / \mathrm{L}$ ) after using the reference method of our laboratory, therefore meaning that the MicroScan system possesses a very low specificity. Concomitantly, the E-test method resulted not to be efficient at determining this possible resistance since its outcomes were MIC $>2 \mathrm{mg} / \mathrm{L}$ in isolates that were susceptible performing microdilution and, at the same time, it showed sensitivity in resistant isolates (false negative or very major errors).

The gathered data brings into question the utility of the colistin wells in these panels from MicroScan system, although maybe their negative predictive value justifies their presence. Therefore, we think that the colistin well should be reviewed in order to determine the possibility of being improved, removed or reinterpreted in the manufacturer's instructions.

For the moment, our recommendation when colistin resistance appears in non-fermenter Gram-negative bacilli, is to always perform a second interpretation, using UMic or other method, following the EUCAST's suggestions. Larger studies involving a higher number of isolates are necessary in order to reach global reliable conclusions. 


\begin{tabular}{|c|c|c|c|c|c|c|c|}
\hline \multirow{3}{*}{$\begin{array}{c}\text { Table } 1 \\
\text { Isolates }\end{array}$} & \multicolumn{7}{|c|}{$\begin{array}{l}\text { Susceptibility to colisitin in Pseudomonas aeruginosa and } \\
\text { Acinetobacter baumannii clinical isolates. }\end{array}$} \\
\hline & \multirow[b]{2}{*}{ Microorganisms } & \multicolumn{2}{|c|}{ MicroScan System } & \multicolumn{2}{|c|}{ E-Test System } & \multicolumn{2}{|c|}{ UMic System } \\
\hline & & MIC (mg/L) & $S / R$ & $\operatorname{MIC}(\mathrm{mg} / \mathrm{L})$ & $S / R$ & MIC (mg/L) & $S / R$ \\
\hline 1 & A. baumannii & $>4$ & $\mathrm{R}$ & 2 & $S$ & 0.06 & $S$ \\
\hline 2 & A. baumannii & $>4$ & $\mathrm{R}$ & 2 & S & 0.125 & $S$ \\
\hline 3 & A. baumannii & 4 & $\mathrm{R}$ & 2 & $S$ & 0.5 & $S$ \\
\hline 4 & A. baumannii & 4 & $\mathrm{R}$ & 2 & S & 0.5 & $S$ \\
\hline 5 & P. aeruginosa & $>4$ & $\mathrm{R}$ & 1.5 & $S$ & 0.5 & $\mathrm{~S}$ \\
\hline 6 & P. aeruginosa & 4 & $\mathrm{R}$ & 1 & S & 1 & $\mathrm{~S}$ \\
\hline 7 & P. aeruginosa & 4 & $\mathrm{R}$ & 1.5 & $S$ & 1 & $\mathrm{~S}$ \\
\hline 8 & P. aeruginosa & 4 & $R$ & 1 & S & 1 & $\mathrm{~S}$ \\
\hline 9 & A. baumannii & 4 & $\mathrm{R}$ & 0.75 & S & 1 & $\mathrm{~S}$ \\
\hline 10 & P. aeruginosa & 4 & $\mathrm{R}$ & 1.5 & S & 1 & $\mathrm{~S}$ \\
\hline 11 & P. aeruginosa & $>4$ & $\mathrm{R}$ & 1 & S & 1 & $\mathrm{~S}$ \\
\hline 12 & P. aeruginosa & 4 & $\mathrm{R}$ & 3 & R & 1 & $\mathrm{~S}$ \\
\hline 13 & P. aeruginosa & $>4$ & $\mathrm{R}$ & 1 & S & 1 & $\mathrm{~S}$ \\
\hline 14 & P. aeruginosa & 4 & $\mathrm{R}$ & 2 & S & 1 & $\mathrm{~S}$ \\
\hline 15 & P. aeruginosa & $>4$ & $\mathrm{R}$ & 6 & R & 1 & $\mathrm{~S}$ \\
\hline 16 & P. aeruginosa & 4 & $\mathrm{R}$ & 6 & R & 1 & S \\
\hline 17 & P. aeruginosa & $>4$ & $\mathrm{R}$ & 0.75 & S & 2 & S \\
\hline 18 & P. aeruginosa & $>4$ & $\mathrm{R}$ & 3 & $\mathrm{R}$ & 2 & $S$ \\
\hline 19 & P. aeruginosa & 4 & $\mathrm{R}$ & 3 & $\mathrm{R}$ & 2 & $S$ \\
\hline 20 & P. aeruginosa & 4 & $R$ & 1.5 & $S$ & 4 & $\mathrm{R}$ \\
\hline 21 & P. aeruginosa & 4 & $\mathrm{R}$ & 4 & $\mathrm{R}$ & 4 & $\mathrm{R}$ \\
\hline 22 & A. baumannii & $>4$ & $\mathrm{R}$ & 24 & $\mathrm{R}$ & 32 & $\mathrm{R}$ \\
\hline 23 & P. aeruginosa & $>4$ & $R$ & 2 & $S$ & 32 & R \\
\hline
\end{tabular}

$\mathrm{MIC}=$ minimum inhibitory concentration.

\section{FUNDING}

None to declare.

\section{CONFLICTS OF INTEREST}

The authors declare that they have no conflicts of interest

\section{REFERENCES}

1. Falagas ME, Rafailidis PI, Ioannidou E Alexiou VG, Matthaiou DK, Karageorgopoulos $\mathrm{DE}_{\text {, et }}$ al. Colistin therapy for microbiologically documented multidrug-resistant Gram-negative bacterial infections: a retrospective cohort study of 258 patients. Int J Antimicrob Agents. 2010; 35: 194-9. doi: 10.1016/j.ijantimicag.2009.10.005.

2. Vourli S, Dafopoulou K, Vrioni G, Tsakris A, Pournaras S. Evaluation of two automated systems for colistin susceptibility testing of car- bapenem-resistant Acinetobacter baumannii clinical isolates. J Antimicrob Chemother. 2017; 72:2528-30. doi: 10.1093/jac/dkx186.

3. The European Committee on Antimicrobial Susceptibility Testing and Clinical and Laboratory Standards Institute. Recommendations for MIC determination of colistin (polymyxin E) as recommended by the joint CLSI-EUCAST Polymyxin Breakpoints Working Group. 2016.

4. Jayol A, Nordmann P, André C, Poirel L, Dubois V. Evaluation of three broth microdilution systems to determine colistin susceptibility of Gram-negative bacilli. J Antimicrob Chemother 2018; 73:1272-8. doi: 10.1093/jac/dky012. 